\title{
Prospective study to assess functional outcomes in patients undergoing single bundle arthroscopic reconstruction of primary anterior cruciate ligament tear comparing with bone quadriceps tendon versus hamstring tendon autograft
}

\author{
Gagan Khanna, Raman Attri*, Rohit Sharma \\ Department of Orthopaedics, Sri Guru Ram Das University of Health Sciences, Amritsar, Punjab, India \\ Received: 14 March 2020 \\ Revised: 28 March 2020 \\ Accepted: 31 March 2020 \\ *Correspondence: \\ Dr. Raman Attri, \\ E-mail: attriraman02@gmail.com \\ Copyright: $(\odot$ the author(s), publisher and licensee Medip Academy. This is an open-access article distributed under \\ the terms of the Creative Commons Attribution Non-Commercial License, which permits unrestricted non-commercial \\ use, distribution, and reproduction in any medium, provided the original work is properly cited.
}

\begin{abstract}
Background: Anterior cruciate ligament (ACL) injuries are one of the most common ligament injuries of knee. Arthroscopic ACL reconstruction has become the standard of care for ACL injuries in the active patient. In present study good bone quadriceps tendon (BQT) autograft and hamstring tendon (HT) autograft will be used for primary arthroscopic ACL reconstruction and its data regarding clinical and functional results will be evaluated and compared. Methods: Sixty patients underwent isolated ACL reconstruction. 30 underwent ACL reconstruction with the BQT and 30 with the HT. The same surgical technique, fixation method and postoperative protocol were used in both groups. Functional outcome in terms of International Knee Documentation Committee (IKDC) score, joint stability (anterior drawer, Lachman, pivot shift), anterior knee pain and isokinetic strength was evaluated.

Results: IKDC score in bone quadriceps tendon and hamstring tendon group after 9 months follow up showing no statistical significance. The negative Lachman component was higher in the BQT group than in the HT group. There was no difference between groups in terms of isokinetic strength.

Conclusions: Our study shows no statistically significant difference between both groups.
\end{abstract}

Keywords: Anterior cruciate ligament, Bone tendon quadriceps, Hamstring tendon

\section{INTRODUCTION}

The knee is a synovial hinge type joint with extensive network of ligaments and muscles. The knee joint is largest and complex joint with a number of internal structures and is capable of performing complex and extensive movements. ${ }^{1}$ The anterior cruciate ligament (ACL) is considered the primary passive restraint to anterior translation of the tibia on the femur, and it provides rotational stability to the knee in both the frontal and transverse planes. $^{2-4}$
There is no gold standard for specific graft there is multiple graft options available for the patient undergoing ACL reconstruction. All possess unique advantages and disadvantages and can be compared using various criteria, including biomechanics properties, biology of healing, ease of harvest, associated graft morbidity. The main objective of our study was to assess the functional outcome preoperative and postoperatively on the basis of International Knee Documentation Committee (IKDC) score among patients with ACL tear repaired with hamstrings graft versus quadriceps. 


\section{METHODS}

The study was conducted in orthopedic department of SGRD IMSR Amritsar. Total 60 cases of ACL tear were included for study purpose. The study was conducted for a period of 18 months (January 2018 to June 2019). ACL tear reconstructed in group 1 with bone quadriceps tendon (BQT) and group 2 with hamstrings tendon (HT) autograft. Selection Criterion for study was as follows:

\section{Inclusion criteria}

Patients with complete ACL tear after 3 weeks of injury were considered for surgery and having high demand of knee bending and pivoting activity were included.

\section{Exclusion criteria}

Patients diagnosed with ACL tear associated with degenerative conditions of knee joint. Patients with multiligament injury were excluded.

\section{Surgical procedure}

The patients admitted on OPD basis and inpatient basis were interviewed with their prior consent both before and after the surgery. Patients was followed up at regular intervals of six week, three months and nine months.

\section{Harvesting BQT autograft}

A suprapattelar incision of about $5 \mathrm{~cm}$ long is performed. Skin flap was raised, exposing the entire width of quadriceps tendon and the central portion of the tendon is harvested with a patellar bone block. The quadriceps tendon is prepared and the free end is stitched to facilitate graft passage. The diameter of the graft is measured.

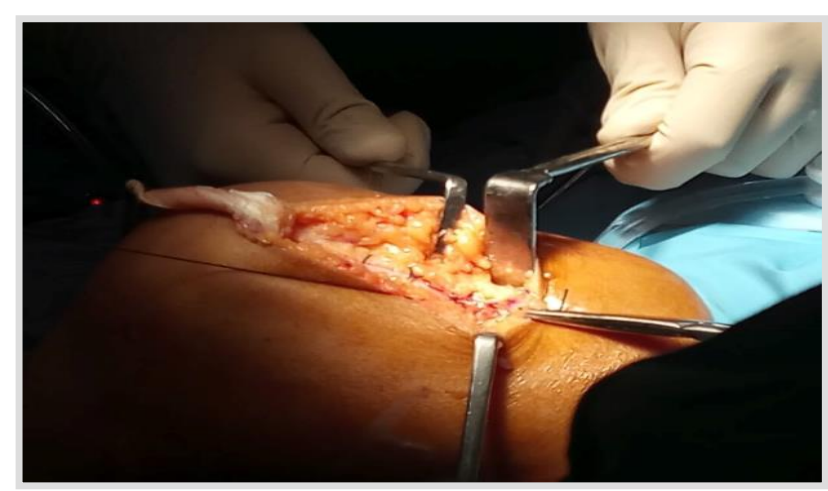

Figure 1: Intraoperative bone quadriceps tendon harvest.

\section{Harvesting semitendinosus or gracillis tendon autograft}

Oblique incision about $3 \mathrm{~cm}$ antero-medially on proximal tibia starting 3-4 cm distal to joint line and 3-4 cm medially to tibial tuberosity was given. Semitendinosus tendon is more horizontal and lies below gracilis. It was pulled with curved clamp or a mixtar and snared with a braided suture, dissection was carried proximally up the thigh.

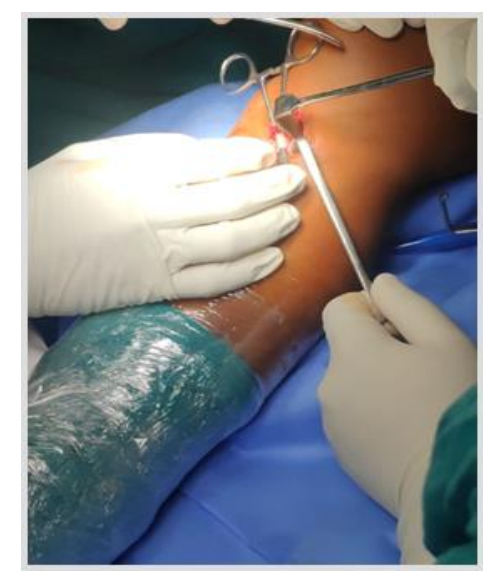

Figure 2: Intraoperative Hamstring tendon graft harvest.

The tibial drill guide should be adjusted to 55 degree angle and the tibial tunnel should be reamed with a cannulated reamer. With the knee maximally flexed for femoral tunnel, an over-the-top guide was inserted through the medial portal and placed in the over-the-top position at the 2-o'clock position for left knees and the 10-o'clock position for right knees.

A brace was given for a period of three weeks. Isometric muscle exercises were started at day one after the operation and gradually progressed on the basis of closed kinetic chain exercises. Indoor cycling was permitted after eight to twelve weeks depending on the patient's tolerability and squatting, jumping, jogging, running was allowed after six months. High demand sports activities were allowed after approximately nine months. Clinical instability tests Lachman test, anterior drawer test, pivot shift test done at each follow up visits.

Data was compiled in MS Excel sheet and statistical analysis was done by SPSS (20.0) version.

\section{RESULTS}

Males were more commonly affected than female patients Right knee joint was more commonly involved than left knee joint. Road side accident was more common cause for ACL injury than twisting injury.

Table 1: Demographic profile of patients.

\begin{tabular}{|lll|}
\hline Variable & $\begin{array}{l}\text { BQT graft } \\
(\text { mean } \pm \text { SD })\end{array}$ & $\begin{array}{l}\text { HT graft } \\
(\text { mean } \pm \text { SD })\end{array}$ \\
\hline Age (in years) & $35.2 \pm 4$ & $33.8 \pm 6$ \\
\hline Sex (male:female) & $27: 03$ & $24: 06$ \\
\hline BMI & $23.2 \pm 2$ & $24.1 \pm 1$ \\
\hline $\begin{array}{l}\text { Time between injury } \\
\text { and surgery (months) }\end{array}$ & $12 \pm 7$ & $14 \pm 5$ \\
\hline
\end{tabular}


Table 2: Comparison of IKDC scores preoperative and postoperative.

\begin{tabular}{|lll|}
\hline IKDC score & BQT graft & HT graft \\
\hline Pre operative & 43.56 & 43.47 \\
\hline Post operative & & \\
\hline 6 weeks & 48.93 & 49.00 \\
\hline 3 months & 65.97 & 64.22 \\
\hline 9 months & 81.55 & 79.92 \\
\hline
\end{tabular}

IKDC score preoperatively in BQT group was 43.56 and post operatively at six weeks, three and nine months was 48.93, 65.97 and 81.55 while in HT group it was 43.47 preoperatively and postoperatively it was 49.00, 64.22, 79.92 at six weeks, three and nine months, which is not significant. $(p>0.05)$ and improved significantly in both the groups at three, six and nine months post operatively $(\mathrm{p}<0.05)$.

\section{Statistical analysis}

The mean IKDC scores in both the groups were analyzed with chi square test with $p$ value 0.05 considered significant. There is no statistically significant difference in IKDC score among at 6 weeks, 3 months as well as 9 months as $\mathrm{p}$ value is $<0.05$. In our study there were no significant difference on comparing the patient's ability to squat, jump, jog, run and go upstairs or downstairs pre operatively and at follow up of six weeks, three and nine months.

\section{DISCUSSION}

In our study statistical analysis shows that there is no statistical significant difference among both groups at 6 weeks, 3 months as well as 9 months as p value is $<0.05$.

There was no significant difference on comparing the patient's ability on squatting, jumping, jogging, running and going upstairs or downstairs pre operatively and at follow up of three, six and nine months $(p>0.05)$ and improved significantly in both the groups at six week, three and nine months.Four studies compared the subjective IKDC score in those with QT and HT. ${ }^{5-10}$ Of those studies, 3 found no significant difference in the subjective IKDC score and 1 found a significantly better subjective IKDC score with QT. ${ }^{5,7,10}$ The overall IKDC score ranged from 80 to 87 with QT and from 78 to 87 with HT. In our study mean diameter of graft used in patients with QT graft was $8.10 \pm 0.51 \mathrm{~mm}$ on femoral side and $9.23 \pm 0.63 \mathrm{~mm}$ on tibial side and for hamstring graft it was $7.64 \pm 0.48 \mathrm{~mm}$ on femoral side and $8.41 \pm 0.57 \mathrm{~mm}$ on tibial side.

Similar results was seen in a study conducted by Todor et al and found that for the QT group the mean graft diameter was $8.57 \pm 0.56(7.5-10) \mathrm{mm}$ on the femoral side and $9.03 \pm 0.63(8-11) \mathrm{mm}$ on the tibial side. In the HT group the grafts had a mean diameter of $7.65 \pm 0.6$ ( 7 to 9 ) $\mathrm{mm}$ on the femur and $8.04 \pm 0.51$ ( 7 to 9 ) $\mathrm{mm}$ on the tibial side. The mean length of the free quadriceps graft was $8.97 \pm 0.58$ (7.5 to 10$) \mathrm{cm}^{11}$

In our study it was observed that among group with Hamstring graft the laxity was grade 0 in 22 pts while 08 had grade 1 laxity. While in pts with quadriceps tendon graft it was absent in 23 pts and 07 had grade 1 laxity. Results are statistically insignificant.Similar results were seen in study conducted by Todor et al. There were no statistically significant differences between the two groups with regard to instrumented Lachman testing, ROM, modified Cincinnati, Lysholm and SF-36 scores. ${ }^{11}$ Similarily, Akoto et al also found no significant difference between the groups was found regarding postoperative knee stability, measured by the Lachman test, Pivot-Shift test or instrumental measurement. ${ }^{12}$

\section{CONCLUSION}

There was no significant difference in functional outcome and knee stability in patients of ACL reconstruction in between Bone quadriceps and HT autografts. There was no difference between groups in terms of isokinetic strength and Range of motion at knee joint.

So, both the grafts can be suitable choice for ACL reconstruction based on surgeon choice and expertise.

Funding: No funding sources

Conflict of interest: None declared

Ethical approval: The study was approved by the institutional ethics committee

\section{REFERENCES}

1. Jenkins DB. Hollinshead's Functional Anatomy of the limbs and Back. Philadelphia: Harcourt Brace \& Company, Jenkins; 1991: 233-239.

2. Kiapour AM, Murray MM. Basic science of anterior cruciate ligament injury and repair. Bone Joint Res. 2014;3:20-31.

3. Butler DL, Noyes FR, Grood ES. Ligamentous restraints to anterior-posterior drawer in the human knee. A biomechanical study. J Bone Joint Surg Am. 1980;62:259-70.

4. Kiapour AM, Wordeman SC, Paterno MV, Quatman CE, Levine JW, Goel VK, et al. Diagnostic value of knee arthrometry in the prediction of anterior cruciate ligament strain during landing. Am J Sports Med. 2014;42:312-9.

5. Cavaignac E, Coulin B, Tscholl P, Nik Mohd Fatmy $\mathrm{N}$, Duthon V, Menetrey J. Is quadriceps tendon autograft a better choice than hamstring autograft for anterior cruciate ligament reconstruction? A comparative study with a mean follow-up of 3.6 years. Am J Sports Med. 2017;45:1326-32.

6. Fischer F, Fink C, Herbst E, Hoser C, Hepperger C, Blank C, Gföller $\mathrm{P}$, et al. Higher hamstring-toquadriceps isokinetic strength ratio during the first 
post- operative months in patients with quadriceps tendon compared to hamstring tendon graft following ACL reconstruction. Knee Surg Sports Traumatol Arthrosc. 2018;26(2):418-25.

7. Hart R, Kucera B, Safi A. Hamstring versus quadriceps tendon graft in double-bundle anterior cruciate ligament reconstruction. Acta Chir Orthop Traumatol Cech. 2010;77:296-303.

8. Joseph M, Fulkerson J, Nissen C, Sheehan TJ. Shortterm recovery after anterior cruciate ligament reconstruction: A prospective comparison of three autografts. Orthopedics. 2006;29:243-8.

9. Kim SJ, Kumar P, Oh KS. Anterior cruciate ligament reconstruction: Autogenous quadriceps tendonebone compared with boneepatellar tendonebone grafts at 2- year follow-up. Arthroscopy. 2009;25:137-44.

10. Kim S-J, Lee S-K, Choi CH, Kim S-H, Kim S-H, Jung M. Graft selection in anterior cruciate ligament reconstruction for smoking patients. Am J Sports Med. 2013;42:166-72.

11. Todor A, Nistor DV, Caterev S. Clinical Outcomes after ACL reconstruction with free quadriceps tendon autograft versus hamstring tendon autograft. A retrospective study with a minimal follow-up two years. Acta Orthop et traumat Turica. 2019;53:18093.

12. Akoto R, Albers M, Balke M, Bouillon B, Hohler J. ACL Reconstruction with Quadriceps tendon graft and Press-fit fixation versus quadruple hamstring graft and interference screw fixation - a matched pair analysis after one year follow up. BMC Musculoskeletal Disorder. 2019;20:109.

Cite this article as: Khanna G, Attri R, Sharma R. Prospective study to assess functional outcomes in patients undergoing single bundle arthroscopic reconstruction of primary anterior cruciate ligament tear comparing with bone quadriceps tendon versus hamstring tendon autograft. Int J Res Orthop 2020;6:511-4. 